\title{
Enfoque Evolutivo del Turismo Rural en Rio Grande do Sul, Brasil
}

\section{Evolutionary Approach of Rural Tourism in Rio Grande do Sul, Brazil}

\author{
Humberto Thomé Ortiz ${ }^{1}$ \\ Eurico de Oliveira Santos ${ }^{2}$
}

RESUMEN - El turismo rural se ha posicionado como una actividad ambivalente que está produciendo resultados variables en el espacio rural brasileño. Con la intención de analizar la evolución del turismo rural en conjunto con la reestructuración productiva del campo, se desarrolló un estudio longitudinal que permite comprender la naturaleza de un medio rural en proceso de transición. Para ello se utilizó un enfoque centrado en los actores rurales, aplicado a nueve propiedades rurales, a través de la realización de cuestionarios en cuatro momentos diferentes (1997, 2002, 2005 y 2011). Se discute que en la Mitad Sur de Rio Grande do Sul, la evolución del turismo narra el debilitamiento de las estructuras productivas tradicionales y la disponibilidad de un patrimonio rural, que responde a una lógica de multifuncionalidad del territorio, pluriactividad de los actores y la emergencia de necesidades urbanas vinculadas con espacios, ambiental y culturalmente, conservados. Se concluye que el turismo rural es una actividad complementaria a las economías campesinas, que permite la reproducción del patrimonio tangible e intangible del medio rural, pero que al mismo tiempo expresa el debilitamiento del tejido social, a través de actividades que no tienen un claro horizonte hacia el futuro.

Palabras clave: Turismo Rural. Reestructuración Productiva. Rio Grande do Sul. Brasil

ABSTRACT - Rural tourism has emerged as an ambivalent activity that has generated mixed results in the Brazilian countryside. The aim of this study was to analyze the evolution of rural tourism and productive restructuring in rural properties in southern of Brazil. For this, a longitudinal study was conducted to characterize the changes towards

\footnotetext{
${ }^{1}$ Doctor en Ciencias Agrarias por la Universidad Autónoma Chapingo, México. Profesor Investigador de Tiempo Completo del Instituto de Ciencias Agropecuarias y Rurales (ICAR) de la Universidad Autónoma del Estado de México. E-mail: humbertothome@hotmail.com

2 Estágio de Pós-doutorado na Universidad Autonoma del Estado de México (UAEMEX) e na Universidade do Aveiro. Doutor en Ciências Agropecuárias e Recursos Naturais pela UAEMEX. Mestre em Extensão Rural pela Universidade Federal de Santa Maria (UFSM). Engenheiro Agrícola pela Universidade Luterana do Brasil (ULBRA). E-mail: metadesul@terra.com.br
} 
multifunctionality of the rural territory. It was used an approach, focusing on rural actors, applied to nine rural properties, using questionnaires at four different times (1997, 2002, 2005 and 2011). It is argued that in the southern of Rio Grande do Sul, the development of tourism shows the weakening of traditional productive structures and the availability of rural heritage, which means a multifunctionality territorial logic, actor's multiactivity and new urban needs related to conserved environmental and cultural spaces. It was concluded that rural tourism is a complementary activity to rural economies, which allows the reproduction of the tangible and intangible heritage of rural space, but at the same time it expresses the weakening of the social structures, through activities that do not have a clear horizon to the future.

Keywords: Rural Tourism. Productive Restructuring. Rio Grande do Sul, Brazil 


\section{INTRODUCCIÓN}

Derivado de la crisis rural del campo latinoamericano, vinculada con la caída de precios de los productos agropecuarios, las migraciones rurales y la desagrarización (FAO, 2014), ha emergido una lógica orientada a buscar oportunidades de diversificación productiva en el contexto de la globalización (AGUILAR; MERINO; MIGENS, 2003). En este sentido, se ha visto en el aprovechamiento turístico de los recursos naturales y culturales, una oportunidad para obtener ingresos complementarios (VEIGA, 2002; THOMÉ-ORTIZ, 2008).

Ello coincide con lo reportado por Zdepski (2001), quien menciona que el turismo rural surge como alternativa para enfrentar la crisis rural, debido a la falta de incentivos para las actividades agropecuarias. De acuerdo, con la Associazione per il Turismo in il Ambiente Rurale - ATAR (1984), las actividades turísticas rurales, además de una fuente de ingresos, suponen un medio para el desarrollo sociocultural del campo, a través de una nueva relación urbano-rural (PÉREZ, 2001).

El objetivo general de este estudio es evaluar la evolución de la actividad turística en nueve propiedades de la Mitad Sur del Estado de Rio Grande do Sul, Brasil, para comprender los procesos de reestructuración productiva en el medio rural. Para ello se aportan datos sobre el perfil de los actores sociales que están desarrollando la actividad, su apego a la tierra y sus características organizativas. Uno de los ejes sustantivos para el logro de este objetivo es documentar la contribución que el turismo hace a las propiedades rurales, desde las perspectivas económica y social.

Específicamente, se buscó diferenciar aquellas propiedades que tienen como insumo turístico fundamental a la cultura rural. El foco de análisis de este trabajo es la perspectiva de los propietarios rurales frente al turismo, por lo que se basa en el trinomio propiedad, propietarios y actividades rurales no agropecuarias.

Se ha elegido la perspectiva del propietario rural dado su potencial explicativo de las transformaciones del medio rural, estando implícito en ella la visión del turista. Se reconoce la importancia que tiene el estudio de la oferta y la demanda en el turismo rural, pero al tratarse de un estudio con enfoque de desarrollo rural, se pone especial atención a los actores rurales, contrario a los estudios con enfoque turístico que se han centrado, mayormente, en el estudio de los turistas (KASTENHOLZ; SANTOS, 2014). 
Se partió del supuesto de que el turismo rural constituye una alternativa de ingreso complementario para las fincas de la Mitad Sur de Rio Grande do Sul y por tanto, se observa su carácter decisivo en la conformación de una nueva ruralidad (GRAMMONT, 2008). Esto lleva a una evolución de las propiedades, dirigida a la multifuncionalidad del territorio (SCHNEIDER, 2003), que es la estrategia que las familias rurales han adoptado para adaptarse a las condiciones macroeconómicas, sin perder la esencia del medio rural, que está basada en la agricultura y la ganadería.

El presente trabajo tiene como objetivo analizar la evolución del turismo rural en el sur de Brasil, visto como un proceso de reestructuración productiva del campo en el contexto de la globalización. Para ello, después de éste apartado introductorio se presenta una aproximación teórica al objeto de estudio, seguida del diseño metodológico de la investigación. Posteriormente, se presentan los resultados y la discusión integradas y se termina con las conclusiones.

\section{MARCO TEÓRICO}

El turismo rural no sólo es una estrategia de desarrollo económico, sino que se concibe como una vía para la preservación del patrimonio sociocultural (OLIVEIRA; DINIZ, 2018). Ello no es una cuestión mecánica sino que parte del marco de referencia de la globalización, en el que los contextos más urbanizados son los que, paradójicamente, encabezan la revaloración de lo rural, asociada con una serie de atributos y ventajas comparativas que el campo adquiere de cara a la ciudad (VEIGA, 2002; AGUILAR; MERINO; MIGENS, 2003).

Lo anterior, es el marco contextual que permite comprender la reestructuración productiva del medio rural brasileño, a través del turismo. Ello tiene múltiples matices que de acuerdo con Carneiro y Maluf (2003) no se pueden interpretar como un proceso de importación mecánica de políticas rurales, con profundas raíces en la experiencia europea, sino que debe leerse desde la especificidad del medio rural Latinoamericano. Dichos aspectos, son los que han despertado el interés por analizar la evolución del turismo rural en el sur de Brasil, como un fenómeno que ilustra ampliamente las transformaciones del campo gaúcho en los últimos años. 
El turismo rural, es un fenómeno que sirve como evidencia empírica de la multifuncionalidad del territorio, al mismo tiempo que da cuenta del estado crítico del espacio rural en búsqueda de alternativas económicas, cuya explicación está íntimamente ligada con las nuevas relaciones que se despliegan entre el campo y la ciudad (THOMÉ-ORTIZ, 2010). Así se confirma con la expansión del turismo rural en diversas partes del mundo.

Cánoves et al. (2004) señalan que el turismo rural se popularizó en Europa desde los años sesenta y se ha convertido en una fuente de ingreso complementario para entre el 2 y el 20\% de las fincas rurales en diferentes países de aquel continente. El número de fincas en España que reciben turistas creció más de seis veces entre 1994 y 2003, aspecto que no sólo tiene que ver con la capacidad de agencia de los actores sociales (SEN, 1985) sino también con un fuerte impulso del turismo como política de desarrollo rural (AGUILAR; MERINO; MIGENS, 2003).

El turismo rural tiene una relación directa con la práctica y contemplación de las actividades desarrolladas en el campo. Esto puede originar una serie de productos y servicios como los "hoteles-hacienda", "pesca y paga", posadas, restaurantes típicos, venta directa de productos locales, artesanías, industrialización y otras actividades de recreación que tienen que ver con la vida cotidiana y con los trabajos rurales (RIVEROS; BLANCO, 2003). Al respecto, Campanhola y Graziano da Silva (2003) definen que estas modalidades turísticas incluyen actividades características de las propiedades, que generan ingresos complementarios al sector agropecuario, las cuales siguen haciendo parte de lo cotidiano, en menor o mayor intensidad, que deben ser entendidas como parte de un proceso de agregación de valor a los productos agrícolas y los bienes no materiales existentes en las propiedades rurales (paisajes, aire puro, historia, etc.), a partir del 'tiempo libre' de las familias con eventuales contrataciones de mano de obra externa.

De acuerdo con Figueroa (2019), el turismo rural se entiende como el ámbito en que los turistas desarrollan sus actividades, más que por el contenido de las tareas que realizan. Es así que la base de este tipo de turismo es el propio ámbito rural, en un sentido amplio. Ello parte de la existencia de un patrimonio natural, histórico-cultural y arquitectónico que despierta el interés de las personas que habitualmente viven en el medio urbano y que están dispuestas a "invertir" parte de su tiempo libre y recursos en 
reencontrarse con sus orígenes, o simplemente, buscan lugares para vacacionar que se encuentren más allá de su tipo de vida habitual.

De acuerdo con Tulik (2003), es más adecuado referirse a la totalidad de los movimientos turísticos que se desarrollan en el medio rural con las expresiones "turismo en el espacio rural o turismo en las áreas rurales", debido a que ello permite la integración de una gran cantidad de productos y servicios desarrollados en el campo. Entonces, el término "turismo rural" es reservado para aquellas actividades que, en mayor grado, se identifican con las especificidades de la vida rural, objeto de estudio de la presente investigación.

Oliveira (2001) afirma que las actividades primarias son el atractivo central del turismo rural, siendo las actividades agropecuarias importantes insumos para el desarrollo de esta estrategia de diversificación productiva. Debido a su capacidad para generar empleos e ingresos adicionales, se entiende la capacidad del turismo rural para propiciar desarrollo en las regiones menos favorecidas. Sin embargo, estas actividades no son concebidas para desarrollarse masivamente y tampoco son una solución definitiva a los graves problemas del sector primario, pero pueden convertirse en un ingreso adicional para los productores. Esto puede contribuir a la mejoría de la calidad de vida rural, respetando la capacidad de carga del ecosistema, con la participación del ciudadano urbano e involucrando los valores socioculturales del campo y la ciudad.

\section{METODOLOGÍA}

Se realizó un análisis comparativo longitudinal del estudio de 9 casos (STAKE, 2000) de propiedades rurales de la Mitad Sur del Estado de Rio Grande do Sul, Brasil, tomando como referencia los periodos de los informes estadísticos elaborados por la SETUR, (Secretaría de Turismo de Río Grande do Sul), de acuerdo con los cuales es posible percibir un lapso de auge y emergencia del turismo rural en el sur de Brasil. Ello se relaciona con procesos históricos de diversificación productiva del campo brasileño, basados en la multifuncionalidad del territorio y la pluriactividad de los actores sociales, los cuales se acentuaron a finales del siglo XX y principios del XXI. Por tal motivo los años 1997, 2002, 2005 y 2011 fueron considerados para obtener información censal de las fincas rurales analizadas, en el marco de un seguimiento a las 
trayectorias evolutivas de los diferentes emprendimientos en el periodo de tiempo analizado. Para Seltiz, Wrightsman y Cook (1987), una forma de clasificación de los tipos de investigación es la que considera la relación con el tiempo, ya que el investigador puede tener interés en estudiar una determinada variable y sus efectos a lo largo de uno o varios periodos establecidos.

El trabajo aborda nueve casos de propiedades que practicaban el agroturismo o el turismo rural, desde el primer periodo de análisis, lo que indica el reciente inicio de la actividad en la región. Estas nueves fincas son las que existían desde 1997 y por tanto son los nueve casos que permiten mejor la realización de un estudio longitudinal en la Mitad Sur de Río Grande do Sul. Para evaluar el desarrollo del turismo y de las fincas, en general, sus propietarios fueron entrevistados en cuatro etapas diferentes, con el objetivo de verificar la evolución de la actividad en un periodo más amplio de tiempo.

Las encuestas fueron de tipo semi-estructurado, incluyendo preguntas abiertas y cerradas. Las respuestas a las preguntas cerradas fueron analizadas mediante estadística descriptiva. Las preguntas abiertas fueron analizadas a través de análisis de contenido (BARDIN, 1977). Se trató de un trabajo centrado en el actor (LONG, 2007), lo que permitió analizar como algunas iniciativas locales, en este caso turísticas, se consolidan como fenómenos globales de la nueva ruralidad (GRAMMONT, 2008). Es una investigación de carácter mixto, que recoge datos cualitativos y cuantitativos, desde la tradición de la sociología rural.

El análisis de los resultados se presenta en cinco partes: 1) localización de las propiedades; 2) características de los propietarios e historia de las propiedades; 3 ) actividad turística; 4) actividad primaria y 5) generación de ingresos complementarios. Se consideró que estos aspectos abordan, de manera amplia, las variables del objetivo de este estudio.

\section{RESULTADOS Y DISCUSIÓN}

\subsection{LOCALIZACIÓN Y TAMAÑO DE LAS PROPIEDADES}

Se identificaron nueve fincas que practicaban turismo rural en 1997, lo que indica el reciente inicio de la actividad. La mayor parte de las propiedades está ubicada 
en las regiones de Campanha (cinco), Centro-Sul (tres) y Frontera Oeste (uno). Cabe hacer notar que cinco fincas de la primera región se encuentran actualmente cerradas. La superficie de las propiedades va de 789 ha a 2,100 ha, con una media de 1,185.2 ha. Según datos de ENGEVIX (1997), la superficie media de las propiedades rurales de la unidad de observación es menor de 50 ha, por lo que en el primer momento del análisis sólo grandes propietarios habían incursionado en la actividad turística.

La distancia entre la capital del Estado (Porto Alegre) y las fincas, va de 125 a $498 \mathrm{~km}$, con la mayor parte de las propiedades en el rango de 300 a $400 \mathrm{~km}$. Debido al cierre de algunas propiedades, la distancia promedio en las fincas, que todavía funcionaban en 2005, se redujo a un rango de 125 a $140 \mathrm{~km}$ de la capital, ya en 2011, la mayor parte de las propiedades se encuentra en el rango de 100 a $200 \mathrm{~km}$. Esto coincide con lo referido por Zimmermann (1996), en el sentido de que el turismo rural es regional y tiene su mayor clientela en un radio de $150 \mathrm{~km}$, o sea, la distancia puede haber sido uno de los factores determinantes del cierre o continuidad de los emprendimientos turísticos. Martínez y Solsona (2000) proponen una clasificación del tiempo de estancia en relación a la distancia, según la cual, cuando el tiempo de viaje es de 1 hora y media y la distancia menor a $150 \mathrm{~km}$, generalmente los huéspedes se quedan el fin de semana, cuando la distancia va de 150 a $300 \mathrm{~km}$, se quedan en días festivos, y cuando la distancia es mayor, durante el período de vacaciones. Algunos estudios (MILBOURNE; KITCHEN, 2014) coinciden con los datos encontrados en los casos analizados, sobre el hecho de que las distancias de la capital del estado hacia las propiedades y la falta de transporte de buena calidad, definen la movilidad en los espacios rurales. Ello implica que el turismo rural se asocia con la proximidad y las distancias entre los atractivos rurales y las fuentes de emisión de turistas, siendo una actividad de proximidad con centros urbanos. 
FIGURA 1 - LOCALIZACIÓN DE LA MITAD SUR DEL ESTADO DE RIO GRANDE DO SUL, BRASIL

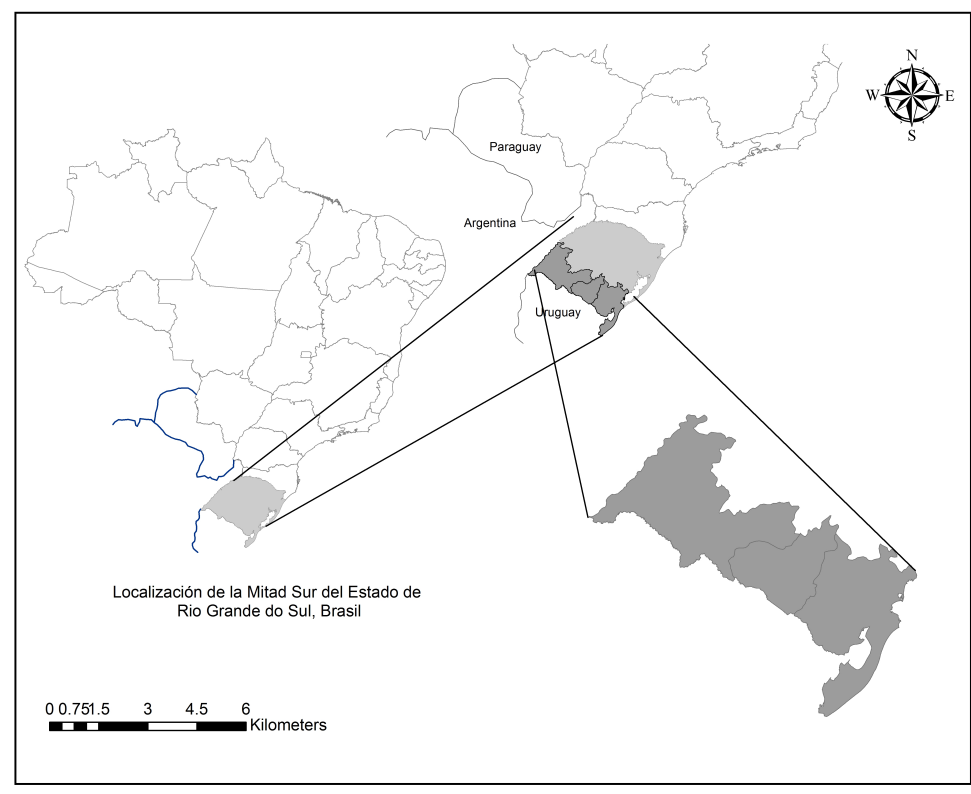

FUENTE: Adaptado de GuiaNet (2019) y Setur (1997)

\subsection{CARACTERÍSTICAS DE LOS PROPIETARIOS E HISTORIA DE LAS PROPIEDADES}

En cuanto al género de los propietarios rurales de los establecimientos investigados, en 2005, seis eran hombres y tres mujeres, en 2011, uno era hombre y dos eran mujeres. En algunas ocasiones, la mujer se convierte en propietaria rural por la muerte de sus padres o su marido, pues recibe la finca como herencia. Ha sido posible observar que las mujeres son quienes mantienen afanosamente estas empresas.

Se observó también que en varios casos la mujer se involucra más que el hombre en la actividad turística, lo que muchas veces implica una duplicación de jornadas, coincidente con lo encontrado en la costa coruñesa por Sparrer (2003). Existe una predominancia de los hombres orientados a las actividades primarias en el campo y de las mujeres a las actividades domésticas. De los encuestados, $66 \%$ cuenta con estudios de tercer grado (bachillerato) o superiores, en Ciencias Agropecuarias y Derecho. Debido a lo anterior, se considera que el nivel de escolaridad es un factor que influye en el desarrollo de los emprendimientos turísticos, el papel de los actores en la consolidación de la actividad concuerda con lo reportado por Komppula (2014) en el caso finlandés. 
Un aspecto interesante es que todos los propietarios, en 2011, vivían en la propiedad, pero poseían una casa en la zona urbana de la ciudad más cercana. Eso consiste en un cambio de comportamiento, orientado por las nuevas movilidades rurales (MILBOURNE; KITCHEN, 2014), al respecto Veiga (2002, p.111) refiere que "un indicador del carácter multisectorial de la renta de los agricultores es el lugar de residencia. El censo agropecuario 1995/1996 mostró la enorme frecuencia con que los agricultores (propietarios rurales) no viven en el establecimiento, sino en la "zona urbana". La escolaridad de los hijos es alta, destacando que de los que poseen estudios universitarios, 100\% no están orientados a disciplinas agropecuarias, presentando un bajo interés por las actividades del campo. Sólo $40 \%$ de los hijos viven en la propiedad, y normalmente son aquellos que no continuaron sus estudios después de la educación media superior.

Los datos dejan dudas sobre la permanencia de las familias en las propiedades durante las próximas generaciones. En cuanto a la historia de la propiedad en la familia, existe una división linear entre las propiedades, dos vienen desde la sexta generación, dos desde la quinta, tres desde la cuarta y tres desde la tercera. Esto parece indicar un apego de las familias por su patrimonio, pero al mismo tiempo, no significa que las mismas permanezcan residiendo o trabajando en el campo. Un aspecto colateral a la permanencia por varias generaciones es el empobrecimiento y deterioro de la infraestructura, evidentes en algunos establecimientos por falta de manutención. La mayoría de las propiedades ha sufrido divisiones a partir de su tamaño original, de entre 4,000 y 60,000 ha antes de ser divididas. Se observa que en todos los casos los propietarios refieren que el turismo es una actividad que contribuye a la manutención de los establecimientos y a evitar su venta.

\subsection{ACTIVIDAD TURÍSTICA EN LAS PROPIEDADES}

La mayor parte de las propiedades (6) inició sus actividades entre 1993 y 1995. Sin embargo, es de resaltar, como se observa en la Tabla 1, que sólo una minoría de las propiedades investigadas seguía con la actividad turística en 2011. 
Humberto Thomé Ortiz; Eurico de Oliveira Santos

TABLA 1 - ACTIVIDAD TURÍSTICA EN LAS PROPIEDADES

\begin{tabular}{lcccccccc}
\hline \multirow{2}{*}{ Actividad turística } & \multicolumn{2}{c}{$1997-1998$} & \multicolumn{2}{c}{$2002-2003$} & \multicolumn{2}{c}{$2005-2006$} & \multicolumn{2}{c}{2011} \\
\cline { 2 - 9 } & Frec. & Porc. & Frec. & Porc. & Frec. & Porc. & Frec. & Porc. \\
\hline Activa & 9 & $100.00 \%$ & 4 & $44.40 \%$ & 4 & $44.40 \%$ & 3 & $33.33 \%$ \\
$\quad$ No activa & - & - & 5 & $55.60 \%$ & 5 & $55.60 \%$ & 6 & $66.67 \%$ \\
\hline Situación Operacional & & & & & & & & - \\
\hline Cierre temporal & - & - & 1 & $20.00 \%$ & - & - & - & - \\
Cierre permanente & - & - & 3 & $60.00 \%$ & 3 & $60.00 \%$ & 3 & $50.00 \%$ \\
Venta & - & - & 1 & $20.00 \%$ & 2 & $40.00 \%$ & 3 & $50.00 \%$ \\
\hline
\end{tabular}

FUENTE: Investigación de campo $(1997,2002,2005$ y 2011)

De las que cerraron, tres lo hicieron de manera permanente. En tres de los casos hubo venta de la propiedad.

TABLA 2 - MOTIVOS PARA EL CIERRE DE LA ACTIVIDAD TURÍSTICA

\begin{tabular}{lcccccc}
\hline \multirow{2}{*}{ Atividad turística } & \multicolumn{2}{c}{$2002-2003$} & \multicolumn{2}{c}{$2005-2006$} & \multicolumn{2}{c}{2011} \\
\cline { 2 - 7 } & Frec. & Porc. & Frec. & Porc. & Frec. & Porc. \\
\hline Poco flujo de turistas & 1 & $20.00 \%$ & - & - & - & - \\
Poca rentabilidad & 2 & $40.00 \%$ & - & - & 2 & $33.33 \%$ \\
$\begin{array}{l}\text { Poca preparación de los } \\
\text { RR.HH. }\end{array}$ & 1 & $20.00 \%$ & - & - & 2 & $33.33 \%$ \\
Estacionalidad del Turismo & 1 & $20.00 \%$ & - & - & - & - \\
$\begin{array}{l}\text { Otras actividades } \\
\text { profesionales }\end{array}$ & 1 & $20.00 \%$ & 2 & $40.00 \%$ & - & - \\
Problemas personales & 1 & $20.00 \%$ & 2 & $40.00 \%$ & - & - \\
Problemas familiares & 1 & $20.00 \%$ & 1 & $20.00 \%$ & 1 & $16.67 \%$ \\
Problemas de salud & - & - & 2 & $40.00 \%$ & - & - \\
NR & 1 & $20.00 \%$ & 1 & $20.00 \%$ & 1 & $16.67 \%$ \\
\hline \multicolumn{1}{c}{ Base } & 5 & - & 5 & - & 6 & - \\
\hline
\end{tabular}

FUENTE: Investigación de campo (1997, 2002, 2005 y 2011)

La Tabla 2 muestra los principales motivos para el cierre de las actividades turísticas, destacándose, en 2011, la poca rentabilidad y poca calificación de la mano de obra para las actividades turísticas. 
TABLA 3 - DIFICULTADES PARA PERMANECER EN LA ACTIVIDAD TURÍSTICA

\begin{tabular}{|c|c|c|c|c|c|c|c|c|}
\hline \multirow[t]{2}{*}{ Dificultades } & \multicolumn{2}{|c|}{$1997-1998$} & \multicolumn{2}{|c|}{$2002-2003$} & \multicolumn{2}{|c|}{$2005-2006$} & \multicolumn{2}{|c|}{2011} \\
\hline & Freq. & Perc. & Freq. & Perc. & Freq. & Perc. & Freq. & Perc. \\
\hline Falta de divulgación & - & - & 1 & $25 \%$ & - & - & - & - \\
\hline $\begin{array}{l}\text { Falta de apoyo } \\
\text { gubernamental }\end{array}$ & - & - & - & - & - & - & 2 & $67 \%$ \\
\hline Distancia/ubicación & - & - & - & - & - & - & 1 & $33 \%$ \\
\hline $\begin{array}{l}\text { Dificultad de } \\
\text { comercialización del } \\
\text { turismo en el espacio rural }\end{array}$ & - & - & - & - & - & - & 1 & $33 \%$ \\
\hline No ve dificultades & 5 & $56 \%$ & 2 & $50 \%$ & - & - & - & - \\
\hline $\begin{array}{l}\text { Falta de mano de obra } \\
\text { calificada }\end{array}$ & - & - & - & - & 2 & $50 \%$ & 1 & $33 \%$ \\
\hline Políticas desfavorables & 1 & $11 \%$ & - & - & - & - & - & - \\
\hline $\begin{array}{l}\text { Falta de preparación de los } \\
\text { servicios }\end{array}$ & 1 & $11 \%$ & - & - & - & - & - & - \\
\hline Falta de incentivo & - & - & - & - & 1 & $25 \%$ & - & - \\
\hline NR & 2 & $22 \%$ & 1 & $25 \%$ & 1 & $25 \%$ & - & - \\
\hline Base & 9 & - & 4 & - & 4 & - & 3 & - \\
\hline
\end{tabular}

FUENTE: Investigación de campo (1997, 2002, 2005 y 2011)

La Tabla 3 discrimina las dificultades para permanecer en la actividad turística. En 2002, la falta de divulgación fue mencionada como dificultad, mientras que en 2005 el nivel de exigencia de mano de obra calificada fue igualmente referido, en 2011, el motivo principal fue la falta de apoyo gubernamental, lo que coincide con lo reportado por Renard-Hubert y Thomé-Ortíz (2010), en el medio rural mexicano.

En relación a la dificultad de acceso, Martínez y Solsona (2000) refieren, que en el contexto europeo cerca de $68 \%$ de las personas que utilizan los servicios de turismo rural hacen su traslado en auto, lo que refleja la importancia de la conservación adecuada de los caminos vecinales (CÁNOVES et al., 2004). Esta situación es similar en las fincas estudiadas, pues está relacionada, según estos autores, a las propias características de la actividad y al perfil de los turistas.

En cuanto al potencial receptivo de las propiedades, la totalidad de las propiedades activas recibe turistas para pasar el día y pernoctar $(100 \%)$, incluyendo alimentación completa. Esto revela que las propiedades tienen una estructura de hospedaje mayor que aquellas que reciben solamente para pasar el día.

Según los propietarios rurales en relación al perfil del turista, la gran mayoría se encuentra entre los 29 y 40 años, son profesionales libres y médicos, casados y acompañados de sus hijos, originarios de varias localidades del Estado. Entre los 
servicios ofrecidos en las propiedades rurales destacan los paseos a caballo, actividades de labranza y piscina, son los más buscados, agregando valor a los demás servicios ofrecidos. Los trabajos de labranza pueden constituir un atractivo importante, pues fueron muy poco referidos en 2005 (25\%), pero en 2011 fueron 100\% referidos, así hubo cambio de preferencia de los turistas. Estas actividades incluyen, según Beni (2008), el manejo del ganado o la colocación de cercas.

También constituyen atractivos para los turistas los "galpones" (almacenes para máquinas e implementos), caminos, cercas, entre otros. Esto coincide con lo reportado por Fleischer y Tchetchik (2005) en Israel, ya que las actividades agropecuarias representan atractivos para los turistas que visitan las fincas. También puede explicarse a partir de lo referido por Almeida y de Souza (2019), según los cuales el turismo rural busca proporcionar descanso y contacto con los valores culturales y patrimoniales, en un contexto diferenciado de la ciudad.

La mayoría de los huéspedes permanece de una a dos noches (67\%) y un menor número $(33 \%)$ por tres o cuatro noches. Esto sugiere varias posibilidades: el turista puede utilizar el lugar para hospedarse en el trayecto de un viaje más largo, o bien, es posible que crea que no haya mucho que hacer en una hacienda. Esta es una de las modalidades referidas por Zimmermann (1996), siendo muy utilizada por representar una mayor rentabilidad, ya que se pueden aprovechar los cuartos para transformar la vivienda rural en una posada. Otra modalidad mencionada por el mismo autor, es el recibir turistas para pasar el día, en la cual se lucra con el valor de los alimentos y la venta de productos.

En cuanto al retorno a la propiedad, la mayoría (67\%) de los huéspedes retorna de una a dos veces al año. Este dato sugiere una preferencia por salidas cortas al campo varias veces al año, en vez de estancias prolongadas. Esta modalidad de vacaciones cortas, pero frecuentes, parece ser una tendencia mundial, en que el estrés diario se acumula rápidamente, siendo necesarias varias paradas a lo largo del año. De acuerdo con Yagüe (2002) se observó una situación similar en España, encontrando preferencia por las distancias cortas y varios viajes por año, lo que puede estar relacionado con la separación de los periodos vacacionales y la aparición de fines de semana largos.

Los propietarios atribuyen el retorno de los huéspedes, de manera general, a la atención personalizada, según Avena (2019), quien recibe huéspedes no vende 
solamente un producto material al cliente, pues es importante que se incluyan la amabilidad, el calor humano, el reconocimiento, la hospitalidad y el cuidado. Siendo los demás motivos: localización, atractivos, contacto con la naturaleza, fuga del estrés y otros repartidos en porcentajes más bajos, ello coincide con lo reportado en México por Thomé-Ortiz (2010).

Existe la posibilidad de realizar actividades de caza y/o pesca en algunas propiedades. Todas las propiedades activas tienen autorización para la pesca. Pero la cacería está bastante restringida y actualmente ninguna de las propiedades tiene autorización para realizarla, debido a una fuerte fiscalización estatal. En relación a la localización, predominó el hecho de que al turista le guste el lugar o región; el contacto con la naturaleza y el contacto con animales.

Los motivos de los propietarios para permanecer en la actividad turística, son principalmente el hecho de gustar de la actividad (50\%), y el éxito de la empresa (50\%). Además de eso, se mencionó que el sector de servicios ya forma parte de la vida en el medio rural $(25 \%)$.

En cuanto a las inversiones en equipamientos para el funcionamiento de la actividad turística, la mayor inversión fue en infraestructura (100\%), en 2002, con una baja en 2005 (75\%), principalmente en reformas y mejorías de la casa, y volviendo al $100 \%$ en 2011. En segundo lugar, aparece la inversión en la compra de muebles y utensilios, que pasó de $33 \%$, en 1997 , a $50 \%$, en 2002 , y $75 \%$, en 2005 , llegando al 100\%, en 2011.

La Tabla 4 describe la percepción del propietario en cuanto a los resultados de la actividad turística en la propiedad. Gran parte de ellos (75\%) percibió los resultados, en 2005, como buenos y una menor proporción (25\%) como satisfactorios. En 2011, la mayoría (67\%) considera los resultados excelentes y lucrativos, el restante (33\%) los considera debajo de lo esperado. Cabe destacar que las respuestas sólo incluyeron a los propietarios de las fincas que permanecen abiertas al turismo. 
TABLA 4 - CÓMO CALIFICA LOS RESULTADOS

\begin{tabular}{|c|c|c|c|c|c|c|c|c|}
\hline \multirow[t]{2}{*}{ Resultados } & \multicolumn{2}{|c|}{$1997-1998$} & \multicolumn{2}{|c|}{$2002-2003$} & \multicolumn{2}{|c|}{$2005-2006$} & \multicolumn{2}{|c|}{2011} \\
\hline & Frec. & Porc. & Frec. & Porc. & Frec. & Porc. & Frec. & Porc. \\
\hline $\begin{array}{l}\text { Excelentes, } \\
\text { actividad } \\
\text { lucrativa }\end{array}$ & 3 & $33.3 \%$ & - & - & - & - & 2 & $66.7 \%$ \\
\hline Buenos & 1 & $11.1 \%$ & - & - & 3 & $75.0 \%$ & - & - \\
\hline Satisfactorios & - & - & - & - & 1 & $25.0 \%$ & - & - \\
\hline $\begin{array}{l}\text { Más positivos } \\
\text { que negativos }\end{array}$ & - & - & 1 & $25.0 \%$ & - & - & - & - \\
\hline $\begin{array}{l}\text { Pequeña, con } \\
\text { tendencia a } \\
\text { mejorar a } \\
\text { mediano y } \\
\text { largo plazo }\end{array}$ & - & - & 1 & $25.0 \%$ & - & - & - & - \\
\hline $\begin{array}{l}\text { Abajo de lo } \\
\text { esperado }\end{array}$ & - & - & - & - & - & - & 1 & $33.3 \%$ \\
\hline $\begin{array}{l}\text { Permiten } \\
\text { continuar }\end{array}$ & - & - & 1 & $25.0 \%$ & - & - & - & - \\
\hline NR & 5 & $55.6 \%$ & 1 & $25.0 \%$ & - & - & - & - \\
\hline Base & 9 & $100.0 \%$ & 4 & $100.0 \%$ & 4 & $100.0 \%$ & 3 & $100.0 \%$ \\
\hline
\end{tabular}

En cuanto al tipo de actividad turística, se constató el predominio de una actividad turística complementaria, pasando del 100\% de las fincas en 1997 al 75\%, en 2002 y 2005 , y, en 2011 , fue el $67 \%$, lo que refiere que el turismo únicamente es un ingreso adicional a sus actividades productivas, pero que cada día cobra mayor relevancia. Esto indica que la actividad permanece como un ingreso importante pero en ningún sentido sustituye a las actividades tradicionales de la propiedad.

\subsection{ACTIVIDAD PRIMARIA EN LAS PROPIEDADES}

La producción de arroz, soya y maíz requiere de grandes inversiones como fertilizantes, semillas, plaguicidas, tractores y cosechadoras, entre otros. El maíz fue el producto más plantado por los propietarios hasta 2005, los agricultores refirieron que es un producto de fácil cultivo. Pero en 2011 hubo un cambio, el producto más plantado fue el arroz. Los cultivos de subsistencia como el fríjol y la papa dulce no aparecen, en las propiedades activas, los propietarios refieren que es más conveniente comprarlos para consumo doméstico que cultivarlos. Los datos revelan que $67 \%$ de los propietarios 
cultiva, pero ninguno lo hace por cuenta propia, existen aparceros (50\%) y arrendatarios $(50 \%)$, que realizan la mayor parte de la actividad agrícola. En cuanto a la maquinaria agrícola, se verifica un cambio relevante. En 1997 todos los propietarios rurales poseían al menos un tractor, mientras que para 2005 sólo 50\% de ellos lo hace, ya en 2011 sólo $33 \%$, y ninguno posee cosechadoras. Datos que en conjunto expresan un debilitamiento progresivo de las actividades agropecuarias, en las fincas que ofrecen servicios turísticos.

Todos los productores depositan sus productos en cooperativas de la región. La principal actividad ganadera es la bovinocultura de corte (100\%), así como la bovinocultura de cría (67\%) y de leche para consumo interno (67\%).

\subsection{GENERACIÓN DE EMPLEO Y RENTA POR SECTOR}

La Figura II muestra la evolución en la generación de empleos, observándose un aumento en el número de empleos generados por finca entre 1997 y 2002 y una disminución para el periodo de 2002 a 2005, y en el periodo de 2005 a 2011 la disminución continúa. Aspecto que refleja que los propietarios de las fincas son quienes se hacen cargo de las actividades turísticas.

FIGURA 2 - GENERACIÓN PROMEDIO DE EMPLEOS POR FINCA

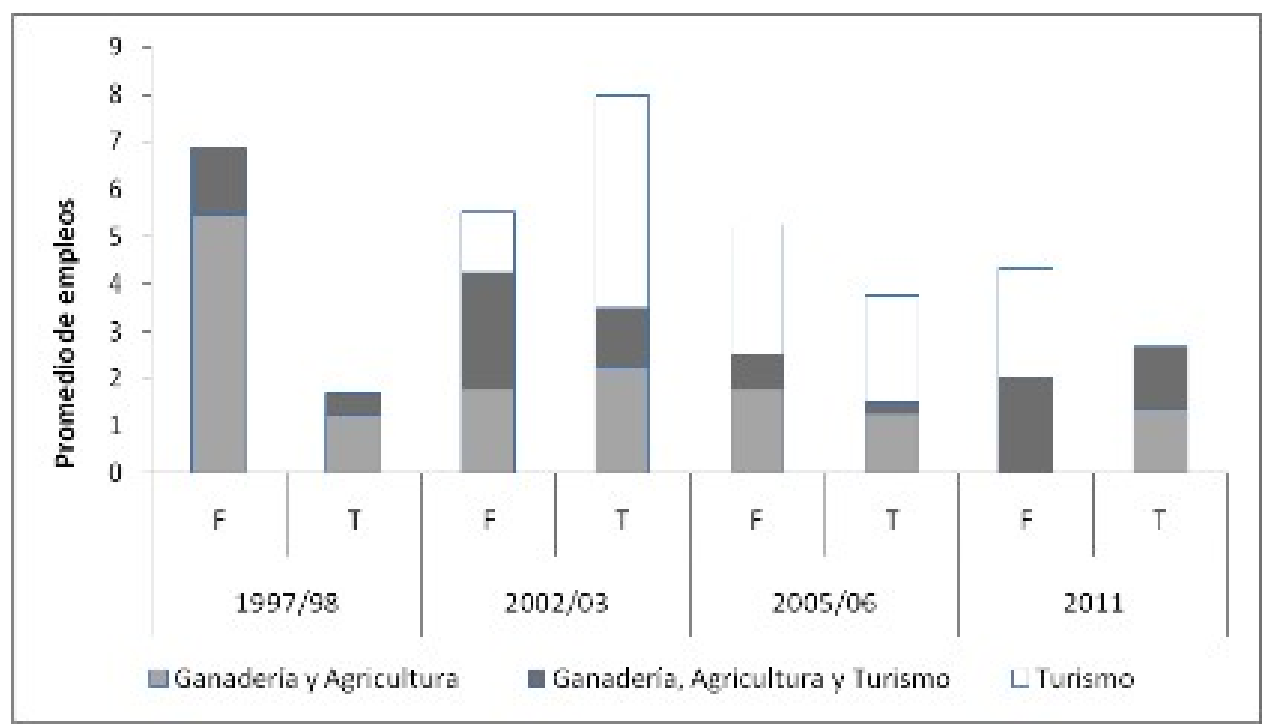

FUENTE: Investigación de campo (1997, 2002, 2005 y 2011) 
El notable aumento de empleos temporales en 2002 puede atribuirse a una situación macroeconómica favorable en ese momento. Sin embargo, el número de empleos generados exclusivamente por la actividad turística mostró un repunte importante en 2005, ocupando en ese año más personas en el sector terciario que en el primario, y observándose una disminución en empleos multifuncionales. Por otra parte, el porcentaje de ingresos derivados del turismo también experimentó un ligero aumento, pasando de $24 \%$ de los ingresos totales, en 1997 , a $26 \%$, en 2002 , a $28 \%$, en 2005 , y a $32 \%$, en 2011 , lo que refleja, en el caso de las fincas que permanecían abiertas a los visitantes, la viabilidad económica de la actividad turística y su capacidad generadora de empleos rurales.

TABLA 5 - DISTRIBUCIÓN PORCENTUAL DE LA RENTA EN LAS PROPIEDADES

\begin{tabular}{lcccc}
\hline \multicolumn{1}{c}{ Sector } & $1997-1998$ & $2002-2003$ & $2005-2006$ & 2011 \\
\hline Primario & 76 & 76 & 73 & 68 \\
Terciario (turismo) & 24 & 24 & 27 & 32 \\
\hline
\end{tabular}

FUENTE: Investigación de campo $(1997,2002,2005$ y 2011)

Los procesos de reestructuración productiva del espacio rural muestran trayectorias evolutivas ancladas a factores estructurales y coyunturales, tal como se ha podido apreciar en el caso brasileño. A continuación se desarrollan las conclusiones del presente estudio.

\section{CONCLUSIONES}

Muchos de los propietarios vivían, exclusivamente, de las ganancias obtenidas en el campo, lo que actualmente es muy complicado debido a las crisis estructurales que presenta el sector agropecuario latinoamericano (FAO, 2014). Sin embargo, más de la mitad de ellos vivía también de otras actividades, lo que tal vez los llevara a no residir exclusivamente en la propiedad. De cualquier modo, en ambos casos, la actividad turística constituyó un ingreso complementario que de acuerdo con Beni (2008), sólo provee un ingreso adicional a la actividad primaria. Las propiedades fueron administradas por los propietarios, indicando un apego al estilo de vida rural. Generalmente, la idea de iniciar en el turismo fue de ellos mismos, revelando una estrategia para la preservación y mantenimiento de sus fincas. Sin embargo, no se 
percibió un interés de los hijos de los propietarios por hacerse cargo de las propiedades ni del negocio turístico. En contraste, la mayoría de los jóvenes tenían estudios en áreas no vinculadas al sector rural.

Dentro de los motivos de los propietarios para entrar al turismo rural, se destacaron los económicos y, principalmente el deseo de aumentar la rentabilidad y agregar valor, lo que coincide con estudios anteriores (BENI; 2008, TULIK; 1997). En cuanto a los motivos de los propietarios para permanecer en la actividad turística, los más frecuentes se relacionaban con el hecho de creer en la actividad turística como fuente de renta adicional a largo plazo, el deseo de convivir con personas diferentes y su contribución al mantenimiento de las propiedades rurales.

Sólo cuatro de las nueve fincas continúan con la actividad turística. La principal dificultad para permanecer en la actividad es la estacionalidad del turismo. Santos (1998) reportó también la falta de mano de obra especializada, la falta de divulgación, no desear trabajar los fines de semana y el hecho de tener que abrir la casa al público. Sin embargo, la mayoría de los propietarios que permanecían en la actividad percibía los resultados de la actividad turística como buenos y satisfactorios, una pequeña parte como excelentes. Todas las fincas han realizado inversiones en infraestructura (mantenimiento y mejora de las casas), siendo el turismo un mecanismo para invertir en el patrimonio de las familias rurales y en la preservación de las propiedades.

Casi todas las propiedades activas recibían huéspedes para pasar el día y para pernoctar, incluyendo los alimentos. La mayoría de los huéspedes permaneció de una a dos noches, retornando a la propiedad de una a dos veces al año, debido principalmente a la calidad de la atención recibida.

El estudio sugiere un apego de los propietarios de las fincas turísticas al medio rural, sin abandonar la actividad agropecuaria, pues el turismo representa su inserción en la pluriactividad. Si bien algunas de las fincas no han sido exitosas, se piensa que la actividad puede ser mejor trabajada y divulgada, expandiéndose y aumentando la calidad del producto turístico ofrecido. Los resultados de este estudio contribuyen para un mayor conocimiento de la actividad turística en la región, a través de la percepción de los propietarios rurales, que son los actores de la transformación productiva del espacio rural. 
Las principales limitaciones de la investigación se relacionan con la disponibilidad de recursos, materiales y humanos, para desarrollar proyectos académicos de más largo aliento, aspecto generalizado en las condiciones para generar conocimiento en Latinoamérica. Ciertamente, la perspectiva exclusiva de los dueños de los emprendimientos turísticos requiere ser contrastada con la de los visitantes, las autoridades locales y la población circundante. Igualmente, la limitación temporal entre 1997 y 2011 debe ser ampliada a periodos más recientes, en la medida en que se pueda acceder a mayores recursos para continuar la investigación. Sin embargo, el análisis de este periodo permite recuperar información clave para entender los procesos de reestructuración económica y productiva del medio rural, donde la turistificación del campo alcanzó un nivel de política global pero que se cristalizó de muy diferentes maneras en la esfera local.

Futuras investigaciones deben incorporar múltiples perspectivas para el análisis del fenómeno turístico en el espacio rural, es necesario trazar las trayectorias evolutivas en líneas de tiempo más largas que se extiendan hasta la actualidad y realizar análisis comparativos con otras zonas de Brasil y el mundo.

\section{AGRADECIMIENTOS}

Los autores agradecen a la Universidad Autónoma del Estado de México por los apoyos de Movilidad Internacional otorgados al Dr. Humberto Thomé Ortiz, que permitieron los trabajos para realizar la presente investigación. .

\section{REFERENCIAS}

AGUILAR, E.; MERINO, D. Y.; MIGENS, M. Cultura, políticas de desarrollo y turismo rural en el ámbito de la globalización. Horizontes Antropológicos, Porto Alegre, v. 9, n. 20, p. 161-183, 2003.

ASSOCIAZIONE PER IL TURISMO IN AMBIENTE RURALE (ATAR). Vademecum dell'operatore di agriturismo. Roma: Terranostra, 1984.

ALMEIDA, J.; DE SOUZA, M. Conceitualização do turismo no espaço rural. Disponível em: http://www.ufsm.br/turismorural. Acesso em: 16 mai. 2019.

AVENA, B. Acolhimento de Qualidade - Fator Diferenciador para o Incremento do Turismo en la Sociedade pos-Industrial. Disponível em:

http:/www.abbtur.com.br/CONTEUDO/trabalhos/trab11.htm. Acesso em: 2 mai. 2019. 
BARDIN, L. Análise de conteúdo. São Paulo: Martins Fontes, 1977.

BENI, M. Análise estrutural do turismo. São Paulo: SENAC, 2008.

CAMPANHOLA, C.; GRAZIANO DA SILVA, J. O novo rural brasileiro - uma análise estadual sul, sudeste e centro-oeste. Jaguariúna: EMBRAPA, 2003.

CÁNOVES, G.; VILLARINO, M.; PRIESTLEY; BLANCO, A. Rural tourism in Spain: an analysis of recent evolution. Geoforum, v. 35, n. 6, p. 765-769, 2004.

CARNEIRO, M.; MALUF, R. Para além da produção: multifuncionalidade e agricultura familiar. Rio de Janeiro: MAUAD, 2003.

ENGEVIX. Plano de reestruturação econômica para a metade sul do Rio Grande do Sul. Porto Alegre: Engevix, 1997.

FIGUEROA, L. Turismo Rural: una nueva alternativa de agronegocios. Disponível em: http://www.promer.cl/getdoc.php?docid=344. Acesso em: 20 mai. 2019.

GUIANET. Guia Cidades. Disponível em: http://www.guianet.com.br/guiacidades/. Acesso em: 10 mai. 2019.

GRAMMONT, H.C. El concepto de nueva ruralidad. In: CORREA, E.P.; QUIJANO, M.A.F.; GRAMMONT, H.C. La Nueva Ruralidad en América Latina, avances teóricos y evidencias empíricas. Colombia: Pontificia Universidad Javeriana, p. 23-44, 2008.

FOOD AND AGRICULTURE ORGANIZATION (FAO). Perspectivas de la agricultura y del desarrollo rural en las Américas: Una mirada hacia América Latina y el Caribe. Costa Rica: CEPAL, FAO, IICA, 2014.

FLEISCHER, A.; TCHETCHIK, A. Does rural tourism benefit from agriculture? Tourism Management, v. 26, n. 4, p. 493-501, 2005.

KASTENHOLZ, E.; SANTOS, E. The profile and motivations of agro-turists in Rio Grande do Sul/Brasil. Pasos, v.12, n. 3, p. 597-609, 2014.

KOMPPULA, R. The role of individual enterpreneurs in the development of competitiveness for a rural tourism destination - A case study. Tourism Management, v.40, p. 361-371, 2014.

LONG, N. Sociología del Desarrollo: una perspectiva centrada en el actor. México: CIESAS, 2007.

MARTÍNEZ, F.; SOLSONA, J. Alojamiento Turístico Rural: Gestión y

Comercialización. Madrid: Síntesis, 2000. 
MILBOURNE, P.; KITCHEN L. Rural mobilities: Connecting movement and fixity in rural places. Journal of Rural Studies, n.34, p. 326 -336, 2014.

OLIVEIRA, C. O sucesso como possível fator de descaracterização de empreendimentos de turismo rural. In: OLIVEIRA, C.; MOURA, J.; SGAI, MARCOS. Turismo no espaço rural brasileiro. Piracicaba: FEALQ, p. 277-283, 2001.

OLIVEIRA, I. D.; DINIZ, F. Turismo e desenvolvimento regional - uma perspectiva do Turismo no Espaço Rural na Serra do Marão, em Portugal. Turismo e Sociedade, Curitiba, v. 11, n. 1, p. 113-127, 2018.

PÉREZ, E. Hacia una nueva visión de lo rural. In: GIARRACA, N. ¿Uma Nueva Ruralidad em América Latina? Buenos Aires: CLACSO, p. 17-29, 2001.

RENARD-HUBERT, M.; THOMÉ-ORTIZ, H. La Ruta de la Sal Prehispánica. Patrimonio Alimentario, Cultural y Turismo Rural en Zapotitlán de las Salinas, Puebla, México. Italy: Departamento de Economía, Universidad de Parma, 2010.

RIVEROS, H.; BLANCO, M. El agroturismo, una alternativa para revalorizar la agroindustria rural como mecanismo de desarrollo local. Lima: IICA/PRODAR, 2003.

SANTOS, E. Turismo rural em fazendas com mais de 1000 ha no estado do Rio Grande do Sul. Porto Alegre: FAMECOS, 1998.

SCHNEIDER, S. A pluriatividade na agricultura familiar. Porto Alegre: Ed. UFRGS, 2003.

SELTIZ, C.; WRIGHTSMAN, L.; COOK, S. Métodos de pesquisa nas relações sociais. São Paulo: McGraw-Hill, 1987.

SEN, A. Well being, agency, and freedom: The Dewey lectures 1984. The Journal of Philosophy, v.82, n. 4, p. 169-221, 1985.

SPARRER, M. Género y turismo rural: ejemplo de la Costa Coruñesa. Cuadernos de Turismo, n. 11, p. 181-197, 2003.

STAKE, R. Case Studies. In: DENZIN, N.; LINCOLN, Y. Handbook of Qualitative Research. London: Sage Publications, p. 134-164, 2000.

SETUR (SECRETARIA DE TURISMO DO ESTADO DO RIO GRANDE DO SUL). Lista das propriedades rurais do Rio Grande do Sul. 1997. Disponível em: www.setur.gov.rs.br. Acesso em: 5 mai. 2019.

THOMÉ-ORTIZ, H. Turismo rural y campesinado, una aproximación social desde la ecología, la cultura y la economía. Convergencia, v. 14, n. 47, p. 237-261, 2008. 
THOMÉ-ORTIZ, H. Turismo en Áreas Rurales, hacia un enfoque de espacios compatibles entre el campo y la ciudad. Artículos y ensayos de sociología rural, n. 9, p. 65-78, 2010.

TULIK, O. Do conceito a las estratégias para o desenvolvimento do Turismo Rural. In: RODRIGUES, A. Turismo: desenvolvimento local. São Paulo: HUCCITEC, p. 136143, 1997.

TULIK, O. Turismo Rural. São Paulo: Aleph, 2003.

VEIGA, J. Ciudades imaginárias: o Brasil é menos urbano do que se calcula. Campinas: Editora Autores Associados, 2002.

YAGÜE, R. Rural tourism in Spain. Annals of Tourism Research, v. 29, n. 4, p.1101$1110,2002$.

ZDEPSKI, F. Aspectos determinantes da oferta para o desenvolvimento do Turismo Rural. In: OLIVEIRA, C.; MOURA, J.; SGAI. Turismo no espaço rural brasileiro. Piracicaba: FEALQ, p. 155-160, 2001.

ZIMMERMANN, A. Turismo Rural: um modelo brasileiro. Florianópolis: Edición Independiente, 1996.

Recebido em: 24-06-2019.

Aprovado em: 07-11-2019.

Versão aprovada para publicação em: 09-11-2019. 